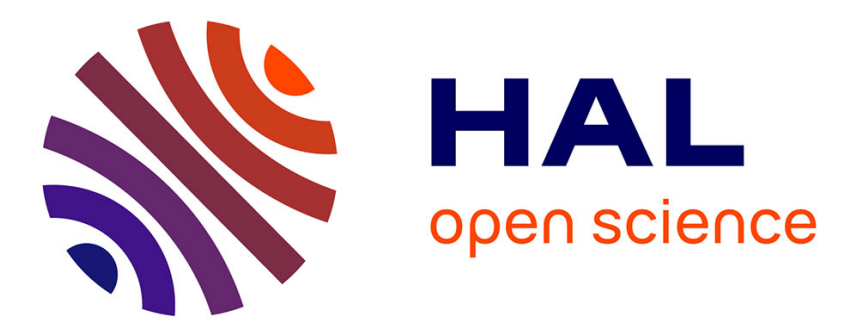

\title{
Configurational mechanics and critical plane approach: concept and application to fatigue failure ananlysis of rubberlike materials
}

\author{
Andri Andriyana, Nicolas Saintier, Erwan Verron
}

\section{To cite this version:}

Andri Andriyana, Nicolas Saintier, Erwan Verron. Configurational mechanics and critical plane approach: concept and application to fatigue failure ananlysis of rubberlike materials. International Journal of Fatigue, 2010, 32 (10), pp.1627-1638. 10.1016/j.ijfatigue.2010.03.004 hal-01005988

\section{HAL Id: hal-01005988 \\ https://hal.science/hal-01005988}

Submitted on 13 Jun 2014

HAL is a multi-disciplinary open access archive for the deposit and dissemination of scientific research documents, whether they are published or not. The documents may come from teaching and research institutions in France or abroad, or from public or private research centers.
L'archive ouverte pluridisciplinaire HAL, est destinée au dépôt et à la diffusion de documents scientifiques de niveau recherche, publiés ou non, émanant des établissements d'enseignement et de recherche français ou étrangers, des laboratoires publics ou privés.

\section{(c)(1)}

Distributed under a Creative Commons Attribution| 4.0 International License 


\title{
Configurational Mechanics and Critical Plane Approach: Concept and application to fatigue failure analysis of rubberlike materials
}

\author{
A. Andriyana ${ }^{\mathrm{a}, \mathrm{b}}$, N. Saintier ${ }^{\mathrm{b}}$, E. Verron $^{\mathrm{c}}$ \\ ${ }^{a}$ Department of Mechanical Engineering, University of Malaya, 50603 Kuala Lumpur, Malaysia \\ ${ }^{\mathrm{b}}$ Arts et Métiers ParisTech, LAMEFIP, Esplanade des Arts et Métiers, 33405 Talence, France \\ ' Institut de Recherche en Génie Civil et Mécanique (GeM), UMR CNRS 6183, École Centrale de Nantes, BP 92101, 44321 Nantes, France
}

From an engineering viewpoint, life time estimation of industrial rubber parts in service is a critical issue. To this end, the present paper outlines recent development and prospect in the application of the Configurational Mechanics, i.e. mechanics of continua expressed intrinsically in the material manifold, and the Critical Plane Approach, entirely formulated in the deformed configuration of the body, to fatigue failure analysis of rubberlike materials. The efficiency of both approaches is discussed and assessed by means of experimental data previously published in the literature.

\author{
Keywords: \\ Crack nucleation \\ Fatigue \\ Rubber \\ Configurational Mechanics \\ Critical Plane Approach
}

\section{Introduction}

The ever-increasing use of rubber in industrial parts, e.g. tires, vibration isolator, seals, medical devices, etc. has kindled considerable interest in the study of their durability in service conditions. Indeed, during design phase of rubber parts, an appropriate method in predicting their fatigue life is highly required. While the development of Finite Element based tools capable of predicting stress and strain histories at each material point of rubber body is now well-established, physical phenomena involved in multiaxial fatigue crack nucleation and growth in rubber materials remain incompletely understood. Consequently, the use of these histories to estimate fatigue life of rubber parts in service remains a critical issue.

In general, the fatigue life of materials involves a period during which cracks initiate in regions that were initially free of observable flaws, followed by a period during which nucleated cracks grow to the point of failure. As emphasized by Mars and Fatemi [28], two approaches corresponding to these two periods are available for predicting fatigue life in rubber: the crack growth (propagation) and the crack nucleation (initiation) approaches. The former can be applied if initial crack shape and position are known. In this case, crack growth is predicted by the calculation of the tearing energy for given specimen under prescribed loading conditions. It is well-adapted to tires problem in which the parts should be able to sustain crack propagation before failure $[20,48]$. In the crack nucleation approach, which is more appropriate for designing engineering parts, the end-oflife is experimentally defined by either a loss of mechanical stiffness [25] or the occurrence of a small crack: $2 \mathrm{~mm}$ for Le Cam [21]; and $1 \mathrm{~mm}$ for André et al. [3], Saintier [36] and OstojaKuczynski [33]. Previous studies showed that both definitions are closely related $[7,11,6,25]$. Indeed, the stiffness decrease can be correlated with the number of cycles required to cause the appearance of a crack of a certain size. It is to note that the crack nucleation approach offers simplicity and familiarity as it is based on quantities that are defined at each material point in the sense of Continuum Mechanics. Hence, no particular consideration on pre-existing crack is needed: the crack initiation location is part of the fatigue life estimation process. The present paper focuses on this approach.

Remark 1. It has to be noticed that the loss of stiffness due to the occurrence of a crack depends on specimen geometry (size effects are obvious when considering stiffness loss as the end-of-life), loading type (a crack of a given size does not have the same effect on the stiffness loss in tension and in torsion due to the stress/ strain gradient) and crack density. A stiffness loss criterion can then encompass a wide variety of crack lengths and densities at the onset of crack initiation. 
The three widely used predictors for fatigue crack nucleation in rubber are the maximum principal stretch ratio $\lambda_{\max }[7,35]$, the maximum principal Cauchy stress $\sigma_{\max }[3,1]$ and the strain energy density $W[35,1]$. Nevertheless, their efficiency in both unifying multiaxial fatigue data and modeling fatigue life reinforcement in rubbers is revealed limited (see [28] and the references herein, and also the recent paper of [1]). Recently, Saintier [36,38] proposed to extend the concept of critical plane, classically applied to metallic materials, to rubber fatigue. To this end, the authors considered both damage and reinforcement mechanisms observed in rubbers. The corresponding mechanisms were then related to the stress histories endured by the material. More precisely, the maximum normal stress acting on the critical plane, defined by the plane experiencing the maximum principal stress over a cycle, was considered to control the fatigue damage evolution. Furthermore, the reinforcement mechanism was related to the minimum crystallinity level on the critical plane during a cycle.

At about the same time, Mars [25,26] proposed a new predictor, referred to as the Cracking Energy Density, by considering that the nucleation of macroscopic fatigue cracks is the consequence of the propagation of microscopic defects initially present in the virgin material. His proposal emphasized two important features: critical plane and energy release rate. Indeed, the author attempted to calculate the portion of the total strain energy density available to be released as microscopic defects grow. In order to determine the orientation of potentially occurring fatigue cracks, the energy that is released on all material planes is calculated. The author concluded that the defect will grow following the plane in which the maximum of energy is released. Moreover, the author compared his proposal against experiments, Linear Elastic Fracture Mechanics, and several cases from the literature. Even if this predictor leads to interesting results, its derivation is questionable since the concept of Cracking Energy Density is not theoretically wellestablished (see the discussions in $[27,46]$ regarding this issue). In an attempt to improve its theoretical foundation using a wellestablished theoretical framework, Verron and Andriyana [46] adopted the Configurational Mechanics theory; more precisely authors related the energy released during defects growth to the properties of the configurational stress tensor. Some progress was made in pursuing it $[5,44,45]$. Nevertheless, further works are required in order to fully establish a rigorous connection between energy release rate and this continuum parameter [2].

The present paper attempts to give a comparative study on the efficiency of two methods used in the prediction of fatigue crack nucleation in rubber: the Configurational Mechanics and the Critical Plane Approach. To this end, previously published experimental data are considered: Saintier [36] and Mars [25]. The paper is organized as follows: In Section 2, both approaches are presented: the general context is briefly recalled, then the application to the prediction of fatigue life in rubber is detailed. Section 3 is devoted to the assessment of the efficiency of both approaches: first basic analytical results are established, then, previously published experimental data are considered. Finally, Section 4 closes the paper.

\section{Two recent predictors for fatigue crack nucleation in rubberlike material}

In this section the two recent approaches emphasized in the Introduction are briefly presented. For each approach some generalities and references are first given, then the application to the case of fatigue crack nucleation in elastomers is presented. For more details, the reader can refer to Saintier [36] and Saintier et al. [37,38] for the Critical Plane Approach and to Verron et al. [47], Andriyana and Verron [5], and Verron and Andriyana [46] for the Configurational Mechanics Approach.

\subsection{Critical Plane Approach (CPA)}

\subsubsection{Generalities}

It is well-recognized that under multiaxial loading conditions macroscopic fatigue cracks in metallic materials nucleate and grow in certain privileged planes, called critical planes. The occurrence and propagation of these cracks are assumed to be due to the stress, strain or/and energy histories acting on the considered planes.

The critical plane, i.e. the one in which the crack will develop, can be determined by identifying the material plane in which a given combination of relevant fatigue damage parameters (expressed in terms of stress, strain and/or energy histories) is maximum. The cornerstone of this approach is the reduction of a multiaxial stress state to an equivalent uniaxial one which combines normal and in-plane quantities (stress, stress or energy) in a fixed plane. Then the resulting equivalent quantity is related to the number of cycles to failure.

The first proposals were founded on the reduction of the Cauchy stress tensor (see for example the pioneering work of Stanfield [39] and the paper of Findley [12] who first introduce the word 'critical'). More recent studies extended the stress-based criteria by considering strain tensor or strain energy density for the derivation of the equivalent mechanical quantity.

Nevertheless, the existing critical plane criteria were developed only for metallic materials under small strain loading conditions. A complete overview of related works was recently proposed by Karolczuk and Macha [18].

\subsubsection{Application to rubber fatigue}

While the CPA enjoyed a great deal of success in metals, only few studies consider this approach to predict fatigue failure in rubber materials because multiaxial fatigue loading effects in elastomers, which often undergo large strain, are not yet well understood. As mentioned previously, the first works which explicitly propose to extend the CPA to fatigue of elastomers are due to Saintier [36] and Saintier et al. [37,38]. Their theory is recalled in the following.

The response of natural rubber subjected to uniaxial fatigue loading conditions exhibit two opposite trends. For negative loading stress ratio $R=\sigma_{\min } / \sigma_{\max }$ and for fixed stress amplitude, fatigue life decreases as the mean stress increases [7,38]. This tendency is reversed as soon as $R$ becomes positive: fatigue life improves under non-relaxing tension cyclic loading. Even if the physical origin of this reinforcement is not completely well-established, it is often attributed to strain-induced crystallization (see for example $[7,13,3])$.

In order to incorporate both fatigue damage and cyclic reinforcement mechanisms into the prediction of fatigue crack nucleation in rubber, Saintier et al. [38] proposed a Cauchy stressbased predictor, denoted $\Phi$, defined by:

$$
\Phi=\frac{\Phi_{\text {damage }}}{1+\Phi_{\text {reinforcement }}},
$$

where $\Phi_{\text {damage }}$ (has stress units) and $\Phi_{\text {reinforcement }}$ (dimensionless) are two quantities associated with damage and reinforcement mechanisms respectively and based on the Cauchy stress history. For the damage part of the predictor, authors have shown from uniaxial fatigue tests that damage is only governed by the normal stress and that compressive stresses on the critical plane has no effect on the fatigue life. Note that these results are in agreement with those of Cadwell et al. [7]. Hence, damage contribution is simply the maximum principal Cauchy stress endured by the material:

$\Phi_{\text {damage }}=\max _{t=0, t_{c}}\left[\sigma_{I}(t)\right]=\sigma_{I}\left(t_{\max }\right)$, 
where $t_{c}$ is the cycle duration, $\sigma_{I}(t)$ is the largest principal Cauchy stress at time $t$ and $t_{\max }$ is the time at which the maximum value of the largest principal Cauchy stress is reached. Moreover, this choice leads to the definition of the critical plane: it is the plane normal to the principal direction associated with $\sigma_{I}\left(t_{\max }\right)$. This direction, denoted $\mathbf{n}_{\mathrm{CP}}\left(t_{\mathrm{max}}\right)$, defines the position of the critical material plane at time $t_{\max }$. Using the classical Nanson formula and normalizing the vector, the position of this material plane in the reference configuration is:

$\mathbf{N}_{\mathrm{CP}}=\mathbf{n}_{\mathrm{CP}}(t=0)=\frac{\mathbf{F}^{T}\left(t_{\max }\right) \mathbf{n}_{\mathrm{CP}}\left(t_{\mathrm{max}}\right)}{\left\|\mathbf{F}^{T}\left(t_{\max }\right) \mathbf{n}_{\mathrm{CP}}\left(t_{\mathrm{max}}\right)\right\|}$,

and its position at any time of the cycle is given by:

$\mathbf{n}_{\mathrm{CP}}(t)=\frac{\mathbf{F}^{-T}(t) \mathbf{N}_{\mathrm{CP}}}{\left\|\mathbf{F}^{-T}(t) \mathbf{N}_{\mathrm{CP}}\right\|}$.

In these equations the superscripts $T$ denotes the transpose of the tensor and $-T$ denotes the transpose of the inverse tensor.

Considering the major influence of strain-induced crystallization on the reinforcement, Saintier et al. [38] proposed to directly relate the reinforcement contribution $\Phi_{\text {reinforcement }}$ to the crystallinity $X_{c}$ observed in the critical plane determined above:

$\Phi_{\text {reinforcement }}=A \cdot X_{c}$,

where $A$ is a material parameter. When $X_{c}$ is equal to zero, i.e. the material returns to a fully amorphous state despite crystallization upon loading, there is no reinforcement contribution. In this case, the predictor $\Phi$ reduces to the damage contribution. From strain-induced crystallinity measurement by X-ray diffraction, authors proposed the following phenomenological relationship between the crystallinity and the Cauchy stress tensor:

$X_{c}\left(\sigma_{\text {reinf }}\right)=0.3\left[1-\exp \left(-D\left\langle\sigma_{\text {reinf }}-\sigma_{0}\right\rangle\right)\right]$,

in which the scalar 0.3 represents the maximum crystallinity in rubber (about 30\% following [24]), $D$ is a material parameter, $\sigma_{\text {reinf }}$ represents the equivalent reinforcement stress and $\sigma_{0}$ is its threshold value under which no crystallinity takes place to be determined by fitting experimental data. $\langle\cdot\rangle$ denotes the MacCauley bracket defined by:

$\langle x\rangle= \begin{cases}x & \text { if } x>0 \\ 0 & \text { otherwise }\end{cases}$

For more details on strain-induced crystallization in rubber, the reader can consult for example $[41,24,42]$ and the references herein.

In order to derive a relevant expression for $\sigma_{\text {reinf }}$, authors considered that reinforcement takes place when the fatigue crack, and consequently the critical plane, does not completely relax during the cycle. Then, one should first determine the stress state in the critical plane. The normal and shear stresses acting on the critical plane at a given time are:

$\sigma_{n}(t)=\mathbf{n}_{\mathrm{CP}}(t) \cdot \sigma(t) \mathbf{n}_{\mathrm{CP}}(t)$,

and

$\tau_{s}(t)=\sqrt{\left\|\boldsymbol{\sigma}(t) \mathbf{n}_{\mathrm{CP}}(t)\right\|^{2}-\left[\sigma_{n}(t)\right]^{2}}$,

respectively. Thus, according to the authors, two different cases are possible for reinforcement to occur (see Fig. 1 for illustration):

1. if the critical plane is subjected to tension-tension $\left(\sigma_{n}\left(t_{\min }\right)>0\right.$, left hand side of Fig. 1), then:

$$
\sigma_{\text {reinf }}=\sqrt{\left[\sigma_{n}\left(t_{\min }\right)\right]^{2}+\left[\tau_{s}\left(t_{\min }\right)\right]^{2}}
$$

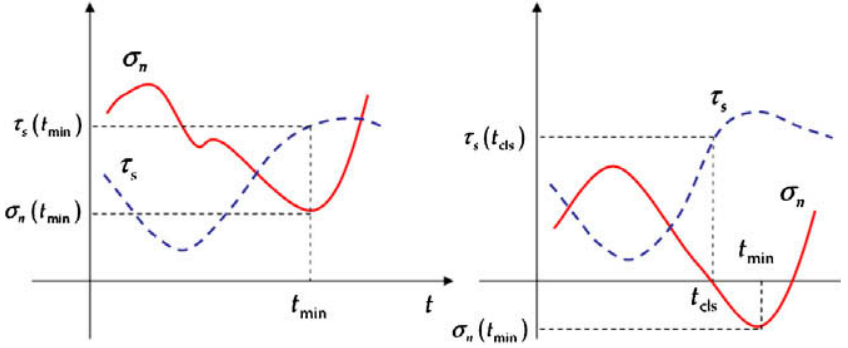

Fig. 1. Arbitrary evolution of normal and shear stresses acting on the critical plane: tension-tension (left) and tension-compression (right).

2. otherwise if it is subjected to tension-compression $\left(\sigma_{n}\left(t_{\min }\right) \leqslant\right.$ 0 , right hand side of Fig. 1 ), i.e. a sort of crack closure effect, then the equivalent reinforcement stress is determined at crack closure time $t_{\mathrm{cls}}$ as follows:

$$
\sigma_{\text {reinf }}=\tau_{s}\left(t_{\mathrm{cls}}\right) \text {. }
$$

In summary, the Critical Plane predictor (referred to as the CPApredictor in the following) is defined by Eqs. (1)-(11) and three material parameters $A, D$ and $\sigma_{0}$ are required beside those of the constitutive model. The number of cycles to fatigue crack nucelation $N_{f}$ is related to $\Phi$ via a classical power law equation

$N_{f}=\left(\frac{\Phi}{\Phi_{0}}\right)^{\alpha}$,

where $\Phi_{0}$ and $\alpha$ are two additional material parameters fitted from uniaxial tension fatigue data.

\subsection{Configurational Mechanics Approach (CMA)}

\subsubsection{Generalities}

As suggested by Gent et al. [14], Mars [26], Le Cam et al. [22], and Le Cam [21] among others, macroscopic fatigue crack nucleation in rubber corresponds to the propagation of microscopic inhomogeneities, such as micro-cracks, inclusions, and cavities, presumably present in the virgin material.

Taking into consideration such phenomena in the derivation of a macroscopic predictor echoes the concept of energy release rate which is the foundation of Fracture Mechanics. Nevertheless, in the present case, the shape and position of initial defects in rubber parts are not known and the definition of energy release rate is not straightforward.

In fact, a general and efficient way to analyze different kinds of material inhomogeneities within the framework of Continuum Mechanics is provided by the theory of Configurational Mechanics $[31,32]$. The introduction of the Configurational Mechanics dates back to the outstanding works of Eshelby $[9,10]$. While in classical Newtonian Mechanics, attention is focused on physical forces generated by displacements in physical space, i.e. the three-dimensional Euclidean space, Configurational Mechanics deals with a different class of forces, referred to as configurational forces, generated by displacements not in the physical space but in the material space, i.e. the abstract set of particles that constitute the body [43].

Generally, material motion in the physical space induces microstructural changes or rearrangements in the material, e.g. growth of microscopic defects, dislocation or displacement of boundary phases. In order to describe such rearrangements, the balance of physical linear momentum should be completely written onto the material space. Following Maugin [32], it leads to

$\operatorname{Div}_{\mathbf{X}} \boldsymbol{\Sigma}+\mathbf{G}=\mathbf{0}$,

in which $\mathbf{X}$ is the position of the particle in the reference configuration and $\boldsymbol{\Sigma}$ is the configurational stress tensor defined by: 
$\mathbf{\Sigma}=W \mathbf{I}-\mathbf{F}^{T} \mathbf{P}=W \mathbf{I}-\mathbf{C S}$,

and $\mathbf{G}$ is the configurational force vector

$\mathbf{G}=-\left.\frac{\partial W}{\partial \mathbf{X}}\right|_{\operatorname{expl}}$.

In these equations, $W$ is the strain energy density per unit of undeformed volume, $\mathbf{I}$ is the $3 \times 3$ identity tensor, $\mathbf{F}$ and $\mathbf{C}$ are respectively the deformation gradient and the right Cauchy-Green strain tensors, and $\mathbf{P}$ and $\mathbf{S}$ are respectively the first and second Piola-Kirchhoff stress tensors.

Moreover, in Eq. (15) $\mathbf{G}$ is defined as the negative explicit differentiation of the strain energy with respect to the particle position in the material manifold (see the index $\left.\right|_{\text {expl }}$ ): if the material is homogeneous, $\mathbf{G}=\mathbf{0}$ and the configurational stress tensor satisfies a strict conservation law.

In the majority of studies involving Configurational Mechanics, only configurational forces are investigated through the calculation of path-independent integrals around inhomogeneities. So, configurational stress only appears in the definition of surface tractions, i.e. after contraction with the outward normal of the contour. Most of these works focus on the application of Configurational Mechanics to Fracture Mechanics (see the brief literature surveys proposed by Steinmann [40] and Verron and Andriyana [46]). For the linear theory, the physical significance of the Cartesian components of the configurational stress tensor were identified only recently by Kienzler and Herrmann [19] for small strain problems. Moreover as proposed by Andriyana [4], the definition can be extended to large strain and arbitrary directions. Indeed, considering a particle and supposing that the configuration of its neighborhood changes due to loading conditions, e.g. growth of cavities, movement of dislocations, phases change, etc., then the configurational stress tensor contains the corresponding changes in energy. More precisely, the material unit translation $\boldsymbol{\theta}$ of a material plane, defined by its normal vector $\mathbf{N}$ and containing the particle, will lead to the change in energy $d U$ given by:

$d U=\boldsymbol{\theta} \cdot \mathbf{\Sigma} \mathbf{N}$.

Remark 2. The interpretation of the "material translation" is discussed for example in [19]. In their paper, the authors consider a volume (or area) element having normal $\mathbf{N}$ at the undeformed configuration, called A1 state. Under loading characterized by the stress field, the associated displacement field and the strain energy density, the element occupies a deformed configuration A2. Assume that these fields are separated from each other as if they would be independent. In addition, consider a neighboring element which, in the undeformed configuration B1, is located at an infinitesimal distance $\boldsymbol{\theta}$ (a vector) from the first element. Under the same loading, this neighboring element will occupy a deformed configuration B2. The transformation from A2 to B2 at constant stress field (since the stress field has been separated from the displacement field) is called material translation.

If now this surface is associated with an idealized crack, when $\boldsymbol{\theta}=\mathbf{N}$, it can be seen as Mode 1 in crack growth. Whenever $\boldsymbol{\theta}$ pertains to the crack tip, it can be seen as Mode 2 in crack growth. Further example on the application of the notion of material translation can be found in [44].

\subsubsection{Application to rubber fatigue}

Very recently, several studies consider the theory of Configurational Mechanics to predict rubber fatigue $[47,5,46]$. Their developments were motivated by the studies focused on the energy release rate of microscopic defects in rubber $[34,16,23,48]$ and more especially the work of Mars [26] who attempted to determine the por- tion of the total strain energy density that is available to be released as microscopic defects grow under multiaxial fatigue loading conditions. In fact his concept of energy release rate was rationalized in the framework of Configurational Mechanics.

This approach is briefly recalled in the following. For additional details, the reader can refer to Verron and Andriyana [46].

In each material point, the calculation of the maximum energy released in the growth of microscopic defects under fatigue loading conditions necessitates the minimization of $d U$ with respect to $\theta$ and $\mathbf{N}$ (Eq. (16)). By supposing that opening and closing of microscopic defects (cavities) in rubber are due to only material normal traction and not due to material shear, the result of this minimization reduces to the smallest eigenvalue of configurational stress tensor $\boldsymbol{\Sigma}$. Thus the predictor is given by:

$\Sigma^{*}=\left|\min \left[\left(\Sigma_{i}\right)_{i=1,2,3}, 0\right]\right|$,

where $\left(\Sigma_{i}\right)_{i=1,2,3}$ are the principal configurational stresses. When one (or more) principal stress is negative, the predictor is strictly positive, and the defect tends to grow and to turn into a plane crack orthogonal to $\mathbf{V}^{*}$, the eigenvector associated with $-\Sigma^{*}$. When the three principal stresses are positive, the material tractions tend to shrink the flaw and the predictor is set to 0 .

In order to account for non-proportional multiaxial loading conditions, authors proposed to accumulate the increment of the configurational stress that contributes to flaw opening. In this case, the previous predictor becomes:

$\Sigma^{*}=\left|\min \left[\left(\Sigma_{i}^{\mathrm{d}}\right)_{i=1,2,3}, 0\right]\right|$,

where $\left(\sum_{i}^{\mathrm{d}}\right)_{i=1,2,3}$ are the eigenvalues of the damage part of the configurational stress tensor $\boldsymbol{\Sigma}^{\mathrm{d}}$. This tensor is obtained by the integration over the cycle of

$\mathrm{d} \boldsymbol{\Sigma}^{\mathrm{d}}=\sum_{i=1}^{3} \mathrm{~d} \Sigma_{i}^{\mathrm{d}} \mathbf{V}_{i} \otimes \mathbf{V}_{i}$,

with

$\mathrm{d} \Sigma_{i}^{\mathrm{d}}= \begin{cases}\mathrm{d} \Sigma_{i} & \text { if } \mathrm{d} \Sigma_{i}<0 \text { and } \mathbf{V}_{i} \cdot \mathbf{\Sigma} \mathbf{V}_{i}<0, \\ 0 & \text { otherwise. }\end{cases}$

$\left(\mathrm{d} \Sigma_{i}\right)_{i=1,2,3}$ and $\left(\mathbf{V}_{i}\right)$ being the eigenvalues and eigenvectors of the configurational stress tensor increment:

$\mathrm{d} \boldsymbol{\Sigma}=\frac{\mathrm{d} \boldsymbol{\Sigma}}{\mathrm{d} \mathbf{C}}: \mathrm{d} \mathbf{C}$

Note that for fully-relaxing proportional loading conditions, the integration over one cycle reduces to the determination of the instantaneous value of the configurational stress tensor corresponding to the maximum strain during cycle. In this case, Eq. (18) reduces to Eq. (17).

Remark 3. The proposed model think of damage as "distributed micro-crack growth". Indeed, it is based on the idea of pre-existing micro-scale flaws that grow under loading without explicitly relate it (in the theoretical derivation) to the stiffness drop. As mentioned in Remark 1, the resulting stiffness drop due to the occurrence of a crack depends on specimen (component) geometry, loading type and crack density.

Remark 4. The motivation of accumulating the configurational stress as a damage measure is discussed in [46]. For simple loading cases including fully-relaxing proportional multiaxial loading, the maximum values of strain components are reached at the same time. Thus, the highest damage level can be simply predicted from the instantaneous value of the configurational stress tensor 
corresponding to the maximum strain. However, for general nonproportional multiaxial loading, the maximum values of strain components are reached at different time. Thus, the resulting damage level depends on the loading history. In order to account for the loading history, it is proposed to accumulate the "damage part" of the configurational stress, which can be regarded as the change of energy associated with the microstructural rearrangement such as micro-crack growth over a cycle [31,32,19].

The unit of the accumulated damage is the one of the configurational stress, i.e. $\mathrm{J} / \mathrm{m}^{3}$ (energy per unit of volume).

In summary, the Configurational Mechanics predictor (referred to as the CMA-predictor in the following) is defined by Eqs. (18)(21) and necessitates no additional material parameters to those of the constitutive model. The reinforcement due to strain-induced crystallization should be accounted for directly into the constitutive equation [5]. Finally, similarly to the CPA-predictor, the number of cycles to fatigue crack nucleation $N_{f}$ is related to $\Sigma^{*}$ via a classical power law equation

$N_{f}=\left(\frac{\Sigma^{*}}{\Sigma_{0}}\right)^{\beta}$

where $\Sigma_{0}$ and $\beta$ are material parameters which should be fitted from uniaxial tension fatigue data.

\section{Example}

A brief recall of the two approaches has been presented. In this section, two experimental data related to fatigue crack nucleation in rubber under different loading conditions are considered, namely Saintier [36] and Mars [25]. To this end, analytical solutions of proportional and non-proportional uniaxial extension/torsion of a hyperelastic tube are firstly recalled. Comparisons between theoretical and experimental results are then presented.

\subsection{Analytical solutions of uniaxial extension/torsion of an incompressible hyperelastic tube}

The complete derivation of the governing equations for the simultaneous uniaxial extension/torsion of a cylindrical hyperelastic tube can be found for example in [15]. Let $(R, \Theta, Z)$ and $\left(\mathbf{e}_{R}, \mathbf{e}_{\Theta}, \mathbf{e}_{Z}\right)$ be the coordinates and the cylindrical unit vectors in the undeformed configuration. Let $(r, \theta, z)$ and $\left(\mathbf{e}_{r}, \mathbf{e}_{\theta}, \mathbf{e}_{z}\right)$ denote their counterparts in the deformed configuration. The deformation of simultaneous uniaxial extension and torsion of a circular tube is given by:

$r=\frac{R}{\sqrt{\lambda}} ; \quad \theta=\Theta+\lambda \tau Z ; \quad z=\lambda Z$,

where $\lambda$ is the stretch ratio and $\tau$ is the twist per unit length.

The corresponding deformation gradient is

$\mathbf{F}=\frac{1}{\sqrt{\lambda}}\left(\mathbf{e}_{r} \otimes \mathbf{e}_{R}+\mathbf{e}_{\theta} \otimes \mathbf{e}_{\Theta}\right)+R \sqrt{\lambda} \tau \mathbf{e}_{\theta} \otimes \mathbf{e}_{Z}+\lambda \mathbf{e}_{z} \otimes \mathbf{e}_{Z}$,

and left and right Cauchy-Green strain tensors can be expressed as

$$
\begin{aligned}
\mathbf{B}= & \frac{1}{\lambda} \mathbf{e}_{r} \otimes \mathbf{e}_{r}+\left(\frac{1}{\lambda}+\lambda^{2} \tau^{2} r^{2}\right) \mathbf{e}_{\theta} \otimes \mathbf{e}_{\theta} \\
& +\lambda^{2} \tau r\left(\mathbf{e}_{\theta} \otimes \mathbf{e}_{z}+\mathbf{e}_{z} \otimes \mathbf{e}_{\theta}\right)+\lambda^{2} \mathbf{e}_{z} \otimes \mathbf{e}_{z},
\end{aligned}
$$

and

$$
\begin{aligned}
\mathbf{C}= & \frac{1}{\lambda}\left(\mathbf{e}_{R} \otimes \mathbf{e}_{R}+\mathbf{e}_{\Theta} \otimes \mathbf{e}_{\Theta}\right)+\tau R\left(\mathbf{e}_{\Theta} \otimes \mathbf{e}_{Z}+\mathbf{e}_{Z} \otimes \mathbf{e}_{\Theta}\right) \\
& +\left(\lambda^{2}+\lambda \tau^{2} R^{2}\right) \mathbf{e}_{Z} \otimes \mathbf{e}_{Z} . \cdot
\end{aligned}
$$

In the hyperelastic framework, the second Piola-Kirchhoff stress tensor is defined as the differentiation of $W$ with respect to the right Cauchy-Green strain tensor C (see for example [17]). Considering that the strain energy only depends on the two first strain invariants $I_{1}$ and $I_{2}$, we have:

$\mathbf{S}=-p \mathbf{C}^{-1}+2\left(W_{1}+W_{2} I_{1}\right) \mathbf{I}-2 W_{2} \mathbf{C}$,

where $p$ is an arbitrary scalar due to the incompressibility kinematic constraint and $W_{i}$ are the partial derivatives of the strain energy with respect to the strain invariants:

$W_{i}=\frac{\partial W}{\partial I_{i}} \quad$ for $i=1,2$.

Using equilibrium equations and the boundary condition which imposes that the external lateral surface is traction free, the expression of pressure $p$ for Neo-Hookean strain energy density $W=C\left(I_{1}-3\right)$ is given by:

$p(R)=2 C \frac{1}{\lambda}+C \lambda \tau^{2}\left(R_{e}^{2}-R^{2}\right)$

where $C$ is material parameter. The Cauchy stress can be expressed as a function of the undeformed radius:

$$
\begin{aligned}
\boldsymbol{\sigma}(R)= & C \lambda \tau^{2}\left(R^{2}-R_{e}^{2}\right) \mathbf{e}_{r} \otimes \mathbf{e}_{r}+C \lambda \tau^{2}\left(3 R^{2}-R_{e}^{2}\right) \mathbf{e}_{\theta} \otimes \mathbf{e}_{\theta} \\
& +2 C \lambda^{\frac{3}{2}} \tau R\left(\mathbf{e}_{\theta} \otimes \mathbf{e}_{z}+\mathbf{e}_{z} \otimes \mathbf{e}_{\theta}\right) \\
& +\left[2 C\left(\lambda^{2}-\frac{1}{\lambda}\right)+C \lambda \tau^{2}\left(R^{2}-R_{e}^{2}\right)\right] \mathbf{e}_{z} \otimes \mathbf{e}_{z} .
\end{aligned}
$$

Furthermore, using Eqs. (26)-(30) and the relationship $\mathbf{S}=$ $J \mathbf{F}^{-1} \boldsymbol{\sigma} \mathbf{F}^{-T}$, the configurational stress tensor reduces to

$$
\begin{aligned}
\boldsymbol{\Sigma}= & C\left(\lambda^{2}+\frac{2}{\lambda}+\lambda \tau^{2} R_{e}^{2}-3\right)\left(\mathbf{e}_{R} \otimes \mathbf{e}_{R}+\mathbf{e}_{\Theta} \otimes \mathbf{e}_{\Theta}\right) \\
& -2 C \tau R\left(\mathbf{e}_{\Theta} \otimes \mathbf{e}_{Z}+\mathbf{e}_{Z} \otimes \mathbf{e}_{\Theta}\right) \\
& +C\left[-\lambda^{2}+\frac{4}{\lambda}+\lambda \tau^{2}\left(R_{e}^{2}-R^{2}\right)-3\right] \mathbf{e}_{Z} \otimes \mathbf{e}_{Z}
\end{aligned}
$$

Finally, the integration of the configurational stress tensor over a fatigue cycle necessitates the determination of its increment:

$\mathrm{d} \mathbf{\Sigma}=\left(\frac{\mathrm{d} \boldsymbol{\Sigma}}{\mathrm{d} \lambda} \dot{\lambda}+\frac{\mathrm{d} \boldsymbol{\Sigma}}{\mathrm{d} \tau} \dot{\tau}\right) d t$

where

$$
\begin{aligned}
\frac{\mathrm{d} \mathbf{\Sigma}}{\mathrm{d} \lambda}= & C\left(2 \lambda-\frac{2}{\lambda^{2}}+\tau^{2} R_{e}^{2}\right)\left(\mathbf{e}_{R} \otimes \mathbf{e}_{R}+\mathbf{e}_{\Theta} \otimes \mathbf{e}_{\Theta}\right) \\
& +C\left[-2 \lambda-\frac{4}{\lambda^{2}}+\tau^{2}\left(R_{e}^{2}-2 R^{2}\right)\right] \mathbf{e}_{Z} \otimes \mathbf{e}_{Z},
\end{aligned}
$$

and

$$
\begin{aligned}
\frac{\mathrm{d} \mathbf{\Sigma}}{\mathrm{d} \tau}= & 2 C \lambda \tau R_{e}^{2}\left(\mathbf{e}_{R} \otimes \mathbf{e}_{R}+\mathbf{e}_{\Theta} \otimes \mathbf{e}_{\Theta}\right)-2 C R\left(\mathbf{e}_{\Theta} \otimes \mathbf{e}_{Z}+\mathbf{e}_{Z} \otimes \mathbf{e}_{\Theta}\right) \\
& +2 C \lambda \tau\left(R_{e}^{2}-2 R^{2}\right) \mathbf{e}_{Z} \otimes \mathbf{e}_{Z}
\end{aligned}
$$

In Fig. 2, the evolutions of the ratio $\Sigma^{*} / \Phi$ as a function of prescribed extension ratio and twist for different loading conditions are presented.

The analytical results were obtained for a cylinder whose radius and height are $12.80 \mathrm{~mm}$ and $5 \mathrm{~mm}$ respectively. The material is assumed to follow Neo-Hookean model with $C=0.36 \mathrm{MPa}$. Note that both $\Sigma^{*}$ and $\Phi$ increase with prescribed deformation but with different rate. Indeed, as shown by the graphs in Fig. 2, $\Phi$ seems to increase at a higher rate than $\Sigma^{*}$ with the applied deformation.

In the case of alternating torsion, more precisions have to be given particularly for the CPA-predictor. Indeed, in this case of fully 

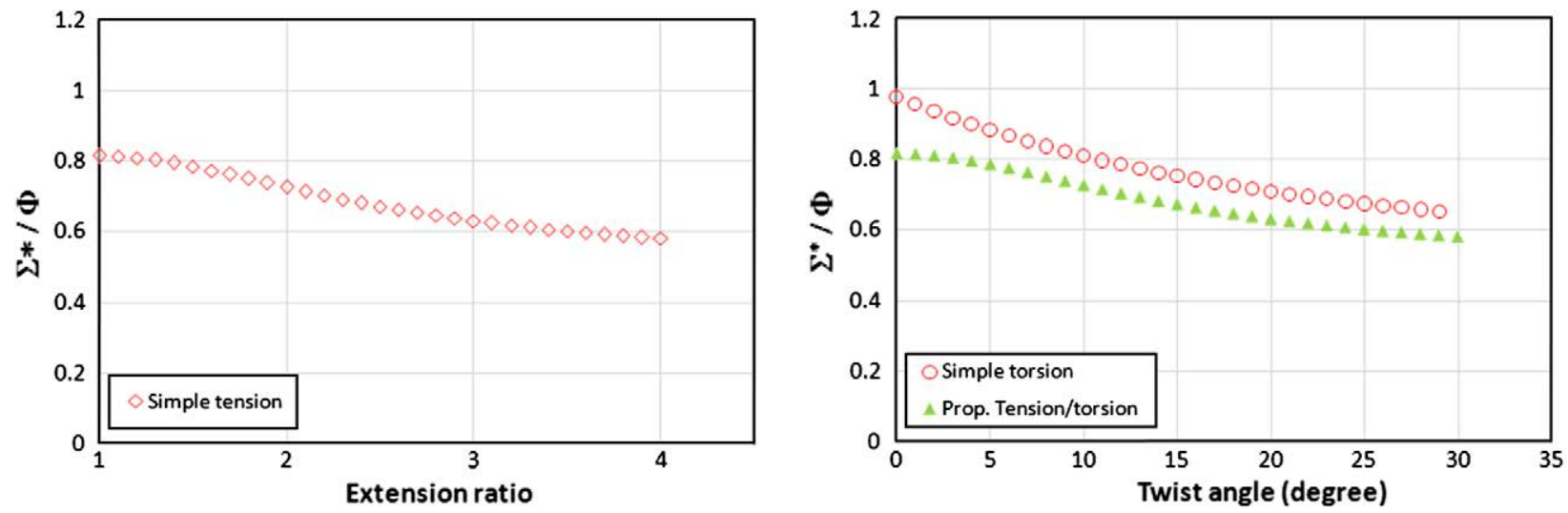

Fig. 2. Evolutions of the ratio $\Sigma^{*} / \Phi$ as a function of prescribed extension ratio (left) and twist (right) for different loading conditions.

symmetrical loadings, two material planes are strictly identical in terms of damage quantity. At a micro-scale, it was shown in $[36,26]$ that for this loading case, micro-cracks alternatively growth along those two planes. In order to correctly assess the fatigue life in such cases, a cumulative law has to be considered. Using a simple Miner cumulative damage law it can easily be shown that the equivalent damage quantity for alternating torsion $\Phi_{\mathrm{AT}}$ can be expressed as a function of that in simple torsion $\Phi_{\mathrm{ST}}$ as follows:

$\Phi_{\mathrm{AT}}=\sqrt[-\alpha]{2} \Phi_{\mathrm{ST}}$

where $\alpha$ is the power law coefficient given in Eq. (12). In the case of CMA-predictor, damage quantities for simple torsion and alternating torsion will naturally differ since $\Sigma^{*}$ is obtained by integration over a cycle (the cumulative process is already done in the formulation). Predictor evolutions for simple torsion and alternating torsion are illustrated in Fig. 3.

The evolutions of theoretical crack angle as a function of prescribed extension ratio or twist for simple tension, simple torsion, alternating torsion and proportional tension/torsion are depicted in Fig. 4. The corresponding angle is obtained by employing Eq. (3) for the CPA-predictor. In the case of CMA-predictor, the crack angle is perpendicular to the eigenvector associated with the smallest principal damage part of the configurational stress tensor $\Sigma^{\mathrm{d}}$ in Eq. (18).

For simple tension and simple torsion loading conditions, both predictors estimate identical theoretical crack angle over a wide range of prescribed deformation. Nevertheless, small differences in the case of proportional tension/torsion are observed. The predicted crack angle in simple tension is equal to zero, i.e. perpendic-

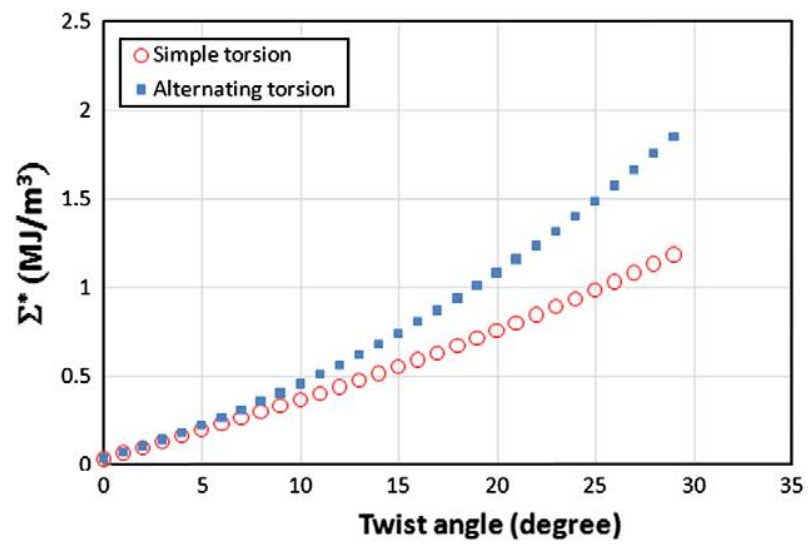

ular to tension orientation and remains unchanged as prescribed extension ratio increases. For simple torsion, the crack angle is $-45^{\circ}$ at small twist (a well-known result in small strain theory) and deviates (decreases) as the prescribed twist increases. Concerning proportional tension/torsion, the maximum prescribed extension ratio is $\lambda_{\max }=1.5$. As shown in the bottom right of Fig. 4, for small twist, the axial (tension) contribution is more important, thus the theoretical crack angle closes to zero. As the prescribed twist increases, the torsion contribution becomes predominant, and the crack angle approaches the one obtained by simple torsion.

Different theoretical crack angles are obtained by the two predictors for alternating torsion loading condition as presented in the bottom left of Fig. 4 .

Indeed, since the use of the CPA-predictor implies that the theoretical crack angle (critical plane) is perpendicular to the eigenvector associated with the maximum value of the principal Cauchy stress over a cycle, two planes are found to experience the same damage quantity. Due to the symmetry of those planes in terms of orientation, the resulting predicted crack angle is zero [36]. In the case of CMA-predictor, it is observed that the theoretical crack angle decreases and approaches zero with the prescribed twist.

\subsection{Comparison with experimental data}

\subsubsection{Experimental data of Saintier [36]}

In this subsection, the experimental data of Saintier [36] are considered. The author investigated fatigue life of a carbon blackfilled natural rubber under different loading conditions: tension/

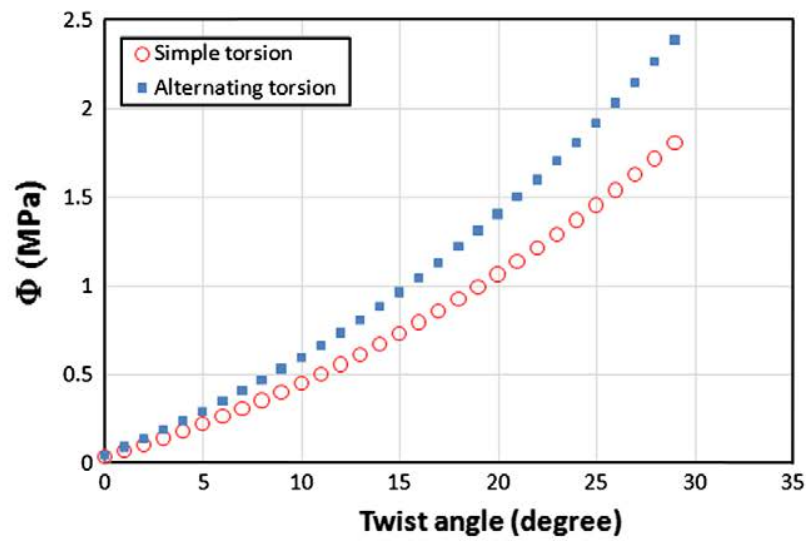

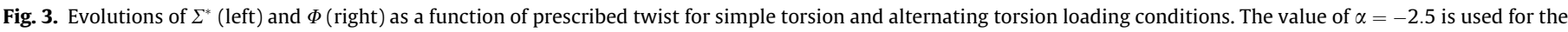
computation of $\Phi$ in the case of alternating torsion. 

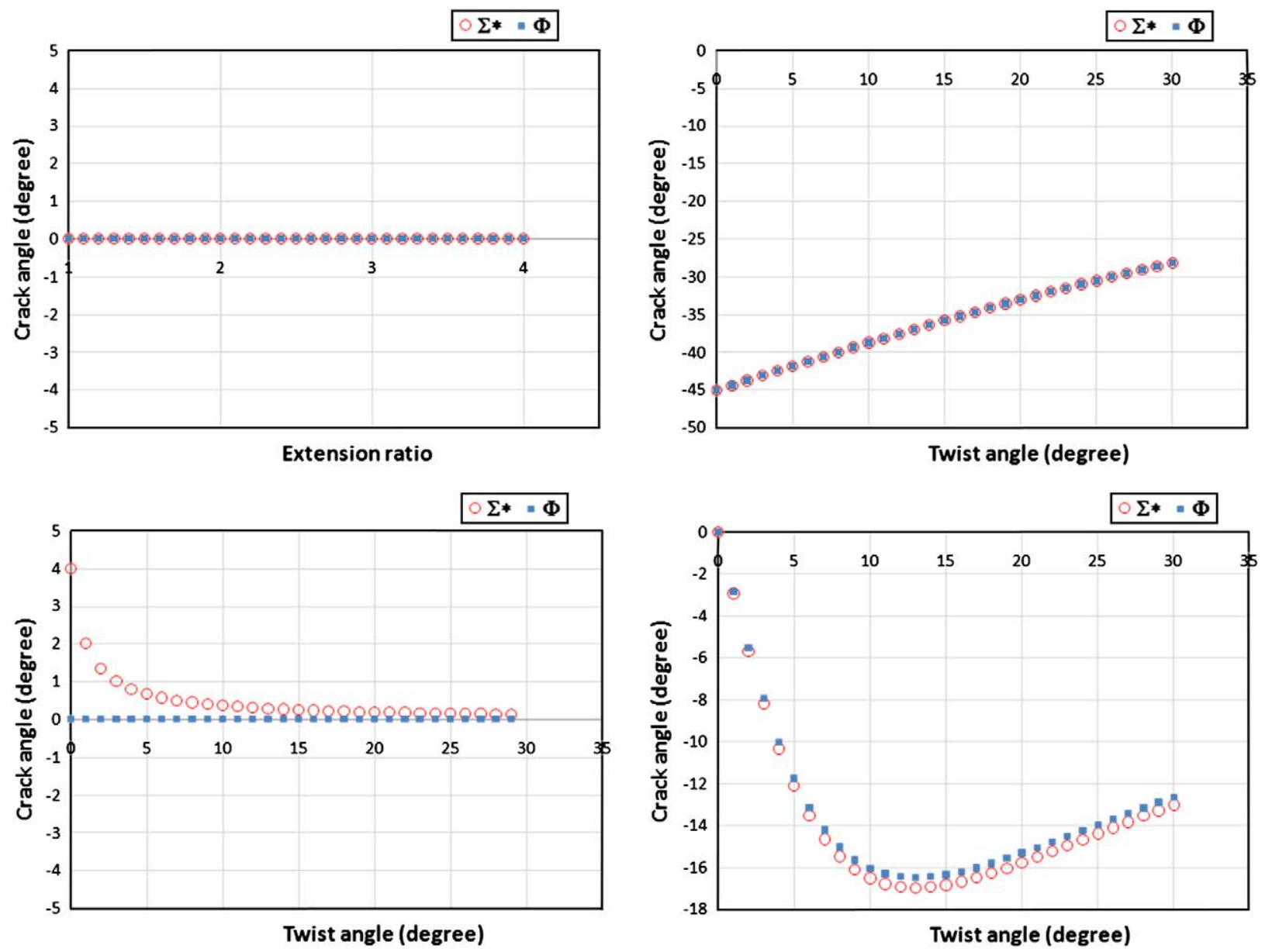

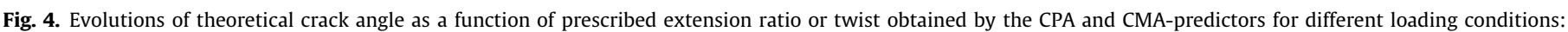
simple tension (top left), simple torsion (top right), alternating torsion (bottom left) and proportional tension/torsion for $\lambda_{\max }=1.5$ (bottom right).

compression with various loading ratios, non-relaxing tension and relaxing/alternating torsion. Three types of specimens were used: Diabolo, AN2 and AN5. All tests were conducted at room temperature and at low frequency $(1 \mathrm{~Hz})$. For each loading condition, the number of cycles corresponds to the occurrence of a $1 \mathrm{~mm}$ long crack was measured. All cracks were observed to nucleate at the specimen surface. The local Cauchy stress at the location of crack nucleation was computed using the Finite Element Analysis (FEA). Note that the author used Mooney-Rivlin series hyperelastic strain energy density for these computations.

In the present study, only experimental data using Diabolo samples are considered (see Fig. 5). Moreover, since numerical implementation of CMA-predictor (particularly its increment over a cycle) on the FE software is still under investigation [46], no local value of accumulated configurational stresses over a cycle on the location of crack nucleation can be obtained using FEA. Therefore, an alternative method to analyze experimental data is proposed here:

1. Diabolo sample is simplified and represented by a simple cylinder whose external radius $R_{e}=12.80 \mathrm{~mm}$ and height $h=$ $10 \mathrm{~mm}$. This geometry corresponds to "effective zone" in the middle of the Diabolo sample as illustrated in Fig. 6.

2. For each loading condition, local Cauchy stresses (maximum and minimum) during a cycle computed using FEA by the author in the original work are considered. To each local Cauchy stress, an equivalent local extension ratio $\lambda_{\max }, \lambda_{\min }$ (and twist per unit length $\tau_{\max }, \tau_{\min }$ ) of the cylinder can be assigned using the corresponding experimental stress-strain curve. Note that in contrast to $\lambda$ and $\tau$, the equivalent twist angle of the cylinder will considerably differ with the real prescribed twist angle of the Diabolo sample during experiment.

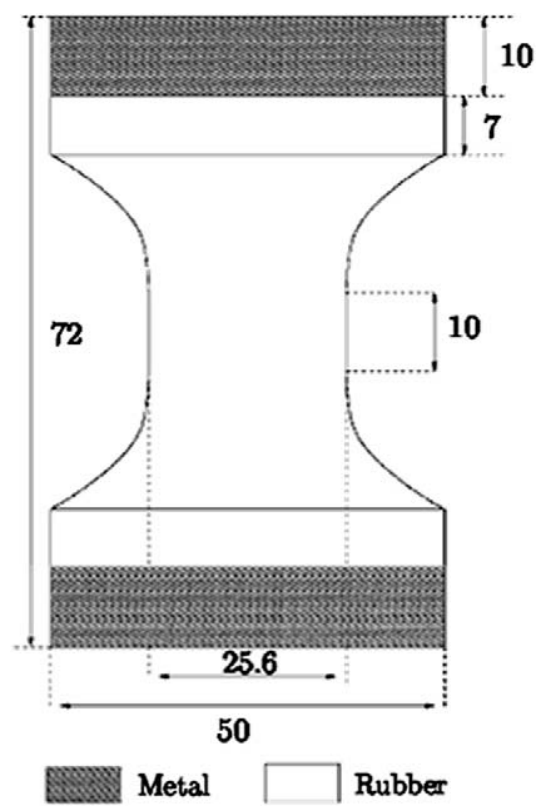

Fig. 5. Geometry of Diabolo samples used for fatigue tests by Saintier [36]. 


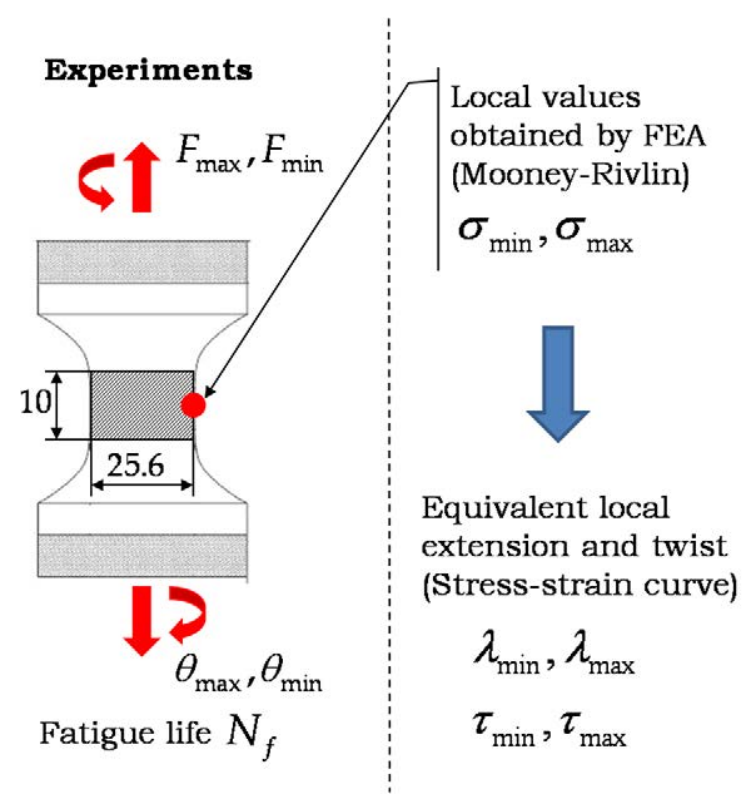

\section{Simplified model}

$\lambda_{\max }, \lambda_{\min }$
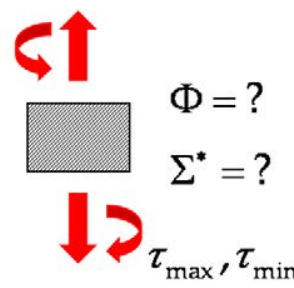

Neo-Hookean

$W=C\left(I_{1}-3\right)$

Fig. 6. Computation of the CPA and CMA-predictors using experimental data of [36].

3. The CPA and CMA-predictors are then computed analytically for these equivalent extension ratio and twist using the Neo-Hookean strain energy density with $C=0.36 \mathrm{MPa}$ fitted from the data of uniaxial tension test.

Fig. 6 summarizes the steps used to evaluate the value of each predictor.

By fitting the uniaxial tension/compression fatigue test data, the following set of material parameters is obtained: $A=7.3, D=$ $0.62, \sigma_{0}=0.17 \mathrm{MPa}, \Phi_{0}=193 \mathrm{MPa}, \alpha=-2.56, \Sigma_{0}=59 \mathrm{MJ} / \mathrm{m}^{3}$ and $\beta=-3.18$. Results are presented in the form of Wöhler curves and fatigue life predictions.

Remark 5. It is important to underline that due to non-relaxing tension cyclic loading (tension-tension), the material exhibits reinforcement mechanism. Here, the corresponding reinforcement is taken into account in the analytical computation of the CPApredictor. The account of reinforcement on the computation of CMA-predictor can be performed by the use of a viscoelastic model [5] which is beyond the scope of the present paper. Hence, reinforcement effect will be neglected from the analytical computation of the CMA-predictor and the results must be interpreted with caution.

In Fig. 7, the Wöhler curves are plotted using the CPA and CMApredictors for different loading conditions: tension-compression, simple torsion, alternating torsion and tension-tension. First, as shown in Fig. 7, the uniaxial and multiaxial (simple torsion) data are well unified using both predictors for low and high fatigue lives.

However, for the CMA-predictor in the case of alternating torsion, one data point slightly deviates from other. Concerning the tension-tension loading in which reinforcement mechanism is observed in the material, both predictors give similar results even if the corresponding reinforcement was not explicitly accounted for in the computation of the CMA-predictor. The comparison between theoretical (predicted) and measured fatigue lives are given in Fig. 8.

In this figure, the diagonal solid line in both graphs represent the case where the predicted and measured lives are identical.
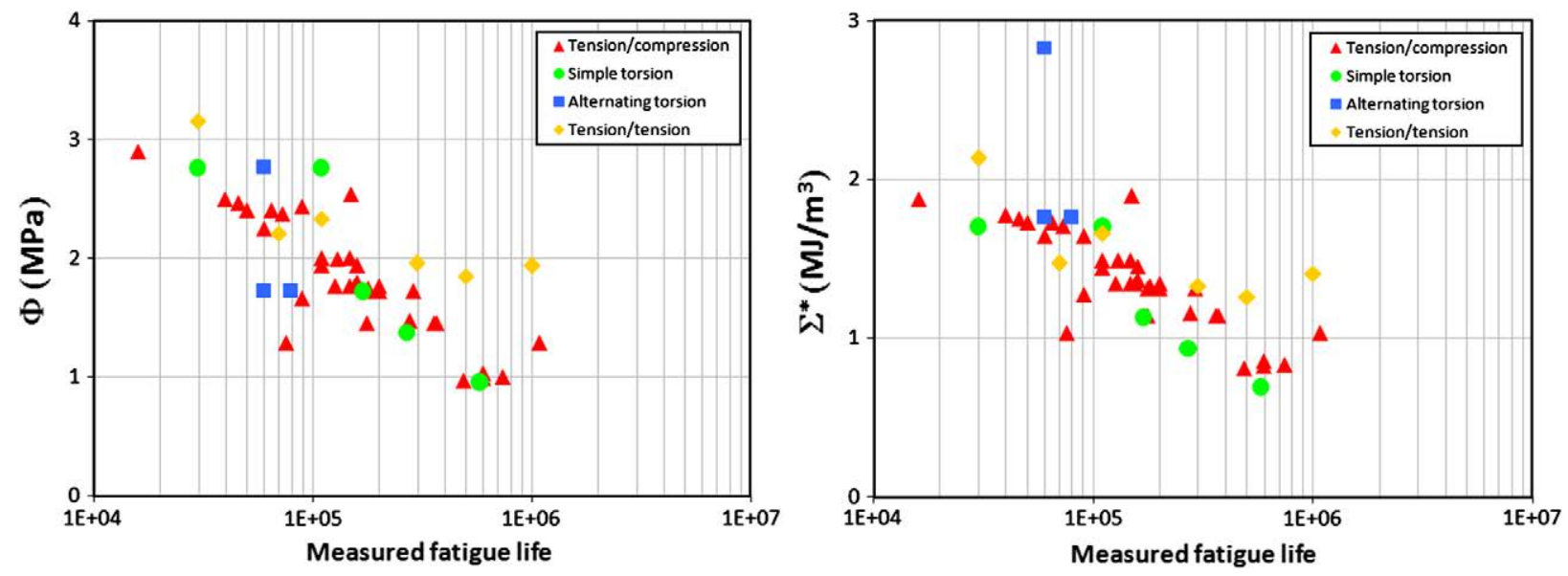

Fig. 7. Comparison of Wöhler curves obtained using the CPA (left) and CMA (right) predictors. Experimental data of [36]. 

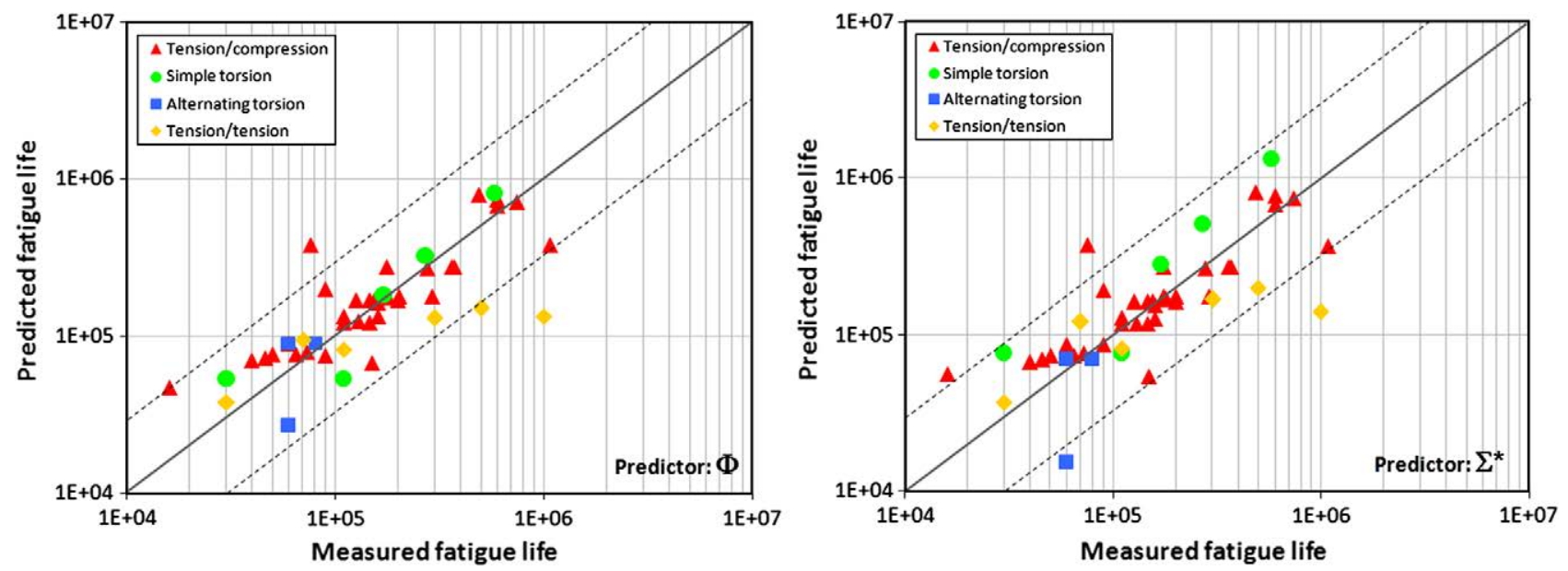

Fig. 8. Comparison between theoretical (computed) and measured fatigue lives using the CPA (left) and CMA (right) predictors. Experimental data of [36].

Moreover, the dashed lines above and below this solid line indicate the case where the predicted fatigue lives are three times higher and lower respectively than the measured one. As shown in Fig. 8, both predictors give a good life estimation. Most of data are located inside the range bounded by the two dashed lines. Finally, it is to note that in the case of simple torsion loading conditions, the differences between the predicted and measured lives obtained by the CPA-predictor are smaller than the one obtained by the CMA-predictor.

\subsubsection{Experimental data of Mars [25]}

In this last example, experimental data of Mars [25] are considered. The author performed a number of uniaxial extension/torsion experiments on carbon black-filled natural rubber for complex multiaxial loading conditions: prescribed force or displacement, proportional and non-proportional, relaxing and alternating. The specimen has a particular geometry: it is a short, hollow cylinder of rubber bonded between two steel mounting rings. Its cross section has a slightly concave surface on the outer diameter and enlarged bonding areas on the top and bottom surfaces. This design was proposed to fulfil, in particular, the following three requirements: to produce a relatively uniform strain field in the sample, to assure that cracks initiate first on the outer surface of the sample and to permit moderate compressive strain without buckling. The test specimen is described and analyzed in detail in [29]. In the following calculations, the sample geometry is simplified and it is considered as a short thick cylinder whose internal radius, external radius and height are respectively $R_{i}=38.10 \mathrm{~mm}, R_{e}=43.18 \mathrm{~mm}$ and $h=6.35 \mathrm{~mm}$. Moreover, only experiments with prescribed displacement (axial and twist) are retained. Test frequencies ranged between 0.5 and $4 \mathrm{~Hz}$, with the higher frequencies at smaller amplitudes (longer lives) to avoid any significant cyclic heating. For a given loading condition, the number of cycle to failure was measured. Note that a $15 \%$ load drop from its stabilized response (defined at 128th cycle) was used as failure criterion.

As conducted in previous examples, to compute the value of each predictor for a given loading, the analytical solution of simultaneous tension/torsion of a hyperelastic cylinder is adopted (see Section 3.1). Following Mars, the material is assumed to obey the Neo-Hookean model with $C=1.5 \mathrm{MPa}$ identified from the first loading monotonic response of uniaxial tension test. It is to note that for the CPA-predictor, reinforcement mechanisms are observed for non-proportional loading conditions. However, since no material crystallinity information is given in the original work, material parameters associated with reinforcement mechanism cannot be identified. Therefore, only proportional loading conditions are considered in the present work. By fitting the uniaxial tension fatigue test data, the following set of material parameters is obtained: $\Phi_{\mathrm{o}}=248 \mathrm{MPa}, \alpha=-2.76, \Sigma_{\mathrm{o}}=111 \mathrm{MJ} / \mathrm{m}^{3}$ and $\beta=-3.23$.

In Fig. 9, the Wöhler curves are plotted using the CPA and CMApredictors for different loading conditions: simple tension $(A)$, simple torsion (B), alternating torsion (C), proportional tension/torsion
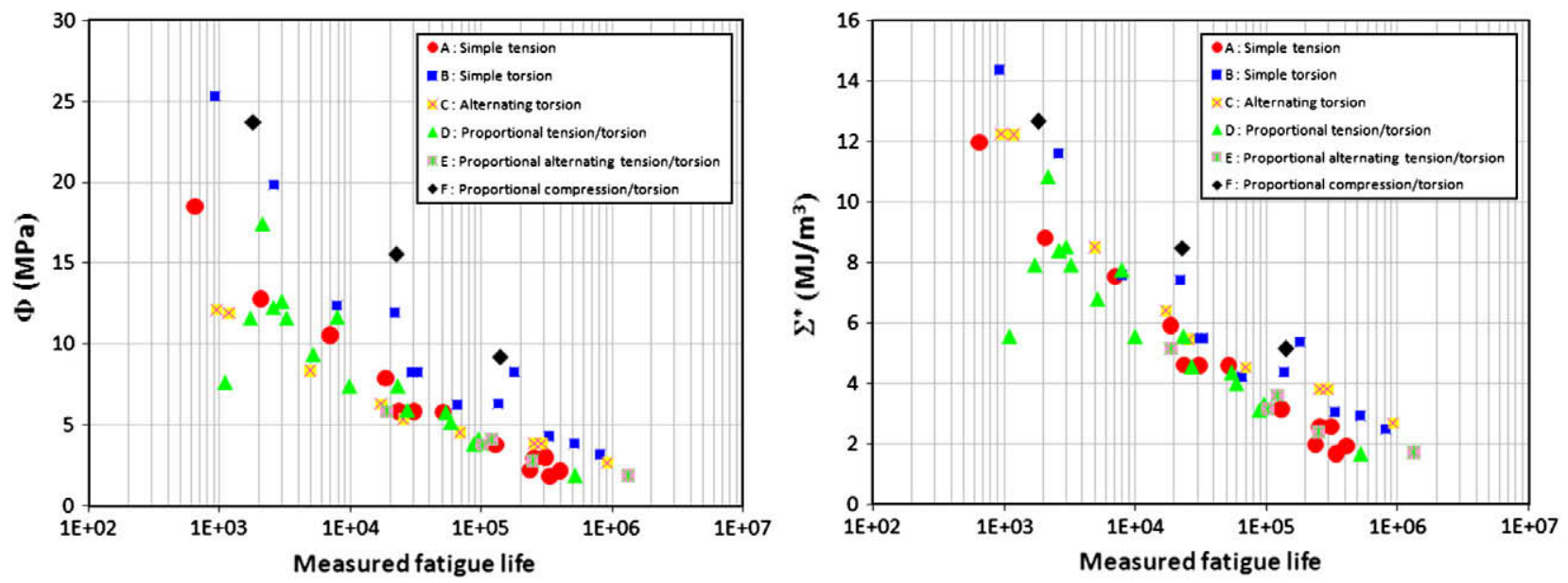

Fig. 9. Comparison of Wöhler curves obtained using the CPA (left) and CMA (right) predictors. Experimental data of [25]. 

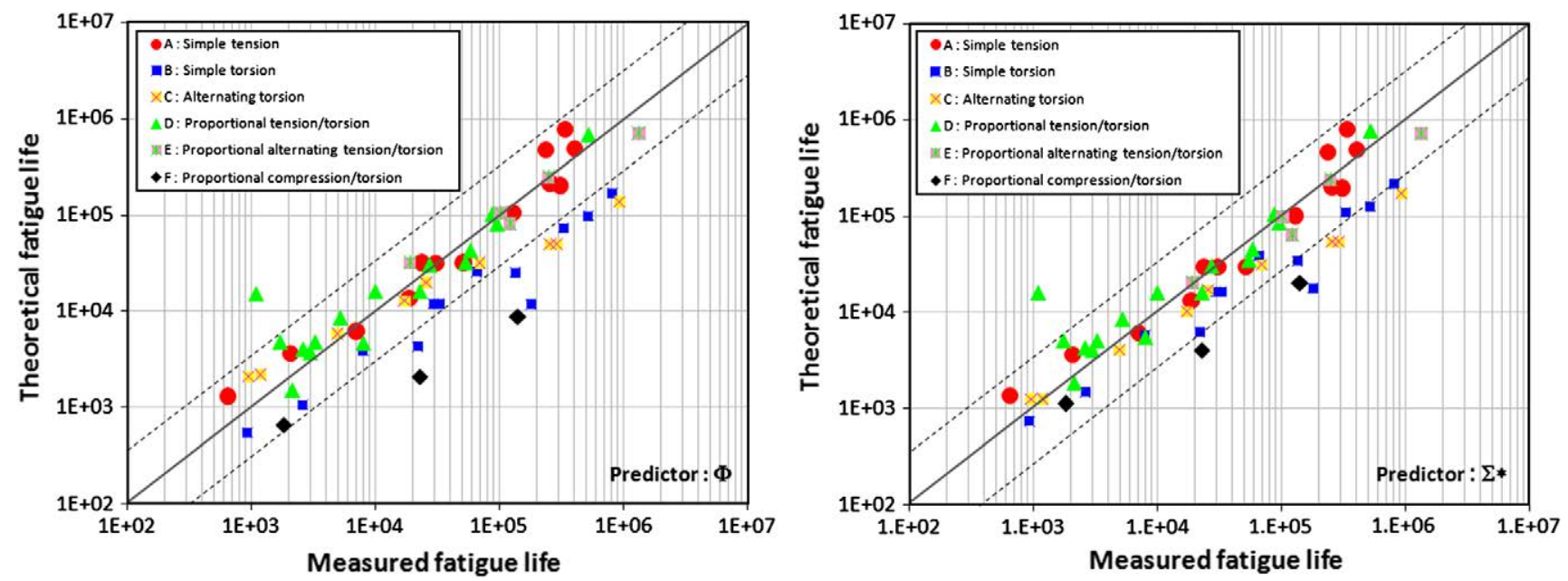

Fig. 10. Comparison between theoretical (computed) and measured fatigue lives using the CPA (left) and CMA (right) predictors. Experimental data of [25].

(D), proportional alternating tension/torsion (E) and proportional compression/torsion (F).

First, as shown in Fig. 9, the data are generally well unified using both predictors for low and high fatigue lives. Nevertheless, both predictors seems to have more difficulties to describe loading conditions where the torsion is predominant, i.e. simple torsion and proportional compression/torsion. In these cases, the CMA-predictor gives slightly better results than the CPA-predictor. The comparison between theoretical (predicted) and measured fatigue lives are given in Fig. 10. In this figure, the diagonal solid line in both graphs represent the case where the predicted and measured lives are identical. Moreover, the dashed lines above and below this solid line indicate the case where the predicted fatigue lives are three times higher and lower respectively than the measured one. As illustrated in Fig. 10, both predictors give a good life estimation. Most of data are located inside the range bounded by the two dashed lines. Finally, it is to note that in the case of simple torsion (B) and proportional compression/torsion (F) loading conditions, the differences between the predicted and measured lives obtained by the CMA-predictor are smaller than the one obtained by the CPA-predictor.

\subsubsection{Discussion}

At this point it is important to make some comments about two issues that have to be highlighted: the end-of-life definition and the constitutive models used for computation. As mentioned in the Introduction, the use of stiffness drop as the end-of-life criteria may lead to different results in terms of crack length and density. Indeed, for a given stiffness drop, the resulting crack length and density depends on the specimen shape and loading type. For instance, consider the specimen geometry used in [25] under torsion and uniaxial tension loading conditions. In the case of torsion, cracks develop at a given angle with respect to the specimen axis. The specimen thickness and the stress gradient (stress decreases as the crack growth within the specimen) induced by the loading limit the extent to which the crack can grow freely. In the case of uniaxial loading, the crack grows in a uniform stress state and is not limited by the specimen geometry (the failure of the specimen can be due to the growth of a single crack). As observed experimentally, the crack density at the end-of-life is higher in torsion than the one in tensile tests. Hence, in term of damage, the crack arrest conditions are not strictly identical. This could explain part of the fact that in the case of Mars's data, numerical fatigue life predictions seems to be systematically smaller than experimental ones.
Another important point that has to be underlined is the choice of the constitutive model. Throughout the present paper, the computation was performed using a simple Neo-Hookean model that captures only a part of the real behavior of the material. In the work of Saintier [36], the material's constitutive behavior was found to be well described by a Mooney-Rivlin model independently of the specimen geometry and the loading condition. A further simplification to a Neo-Hookean model in the present work still gives a good description of the material behavior considering the stress and strain ranges. The material used in [25], however, exhibits a strong stress softening (Mullins effect). Considering the fatigue life ranges, it is found that for Saintier, $80 \%$ of the results are in the range of $\left[8 \times 10^{4}: 10^{6}\right]$ cycles whereas for Mars $80 \%$ of the experimental fatigue life are in the range of $\left[10^{3}: 10^{5}\right]$ cycles. Thus, despite the fact that the materials used in the two works are not the same, the stress/strain levels in Mars's results are much higher than in the case of Saintier. Consequently, the use of a simple model such as a Neo-Hookean to describe the real local stress/ strain response for Mars's material is questionable for at least two reasons. First, the test being performed at high strain level under displacement control, a strong Mullins effect occurs during the fatigue life (it has to be remembered that the constitutive parameter $C$ in the Neo-Hookean model was identified from the first loading monotonic response). Secondly, as underlined by Mars and Fatemi [30], Mars's material behavior under proportional and non-proportional loading deviates from a nonlinear elastic behavior so that the stress-strain behavior may be idealized as a family of stressstrain curves. The particular curve of relevance at any moment depends on the maximum strain previously experienced due to the Mullins effect. Hence, to fully describe and correctly compute the cyclic stress-strain response, a more complex nonlinear viscoelastic model that takes into account the Mullins effect is required. Nevertheless, the establishment (and identification) of such a mod$\mathrm{el}$ is clearly beyond the scope of the present paper. For a review of the Mullins effect, the reader can consult for example the work of Diani et al. [8].

Cyclic stress-strain curves given by Mars and Fatemi [30] for proportional and non-proportional tension-torsion loading indicates that the Mullins effect depends on the loading type. Fig. 11 illustrates the stress softening, defined by the difference between computed (monotonic) and experimental (cyclic) maximum ax$\mathrm{ial} /$ shear engineering stress components at the 128th cycle, as a function of the fatigue life for different loading conditions.

For simple torsion (resp. simple tension) loading, only engineering shear (resp. axial) stress softening are represented. In contrast, 


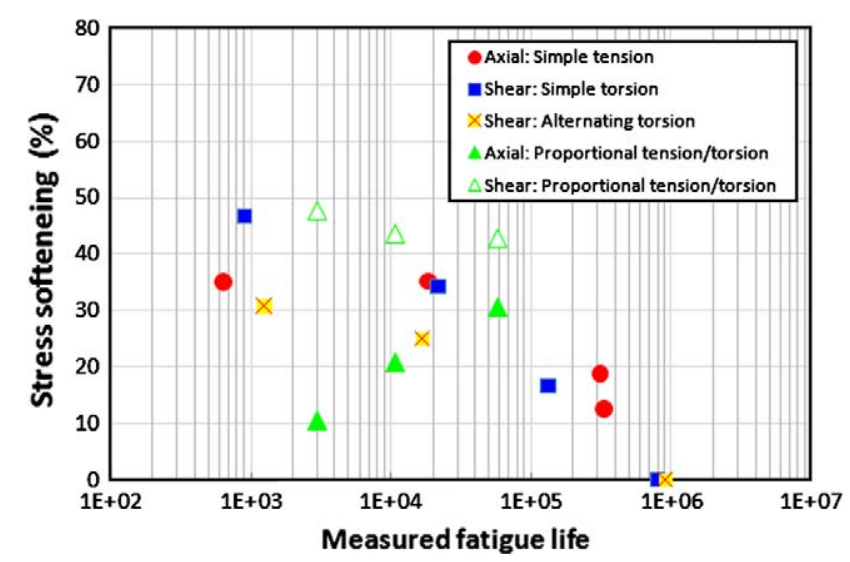

Fig. 11. Stress softening observed in axial and shear engineering stresses for different loading conditions. Experimental data of [25].

for proportional tension/torsion loading, both axial and shear stress softening are given. As shown in this figure, for a given loading condition, the stress softening depends on the fatigue life: it decreases with fatigue life except for the axial stress component of the proportional tension/torsion loading. Moreover, for fatigue lives lower than $10^{5}$ cycles, the scatter in stress softening becomes more pronounced. Indeed, the corresponding softening varies from $10 \%$ to nearly $50 \%$. Finally, from the fatigue prediction point of view, it is to note that while the neglect of the stress softening on the description of material's constitutive behavior may adversely affect the ability of both predictors, in the particular case of simple tension the discrepancies between theoretical and measured fatigue lives are reduced by the fact that the power law in Eqs. (12) and (22) were identified from uniaxial tension fatigue data.

\section{Conclusions}

The present paper attempted to give a brief comparative study on the efficiency of two recent approaches used in the prediction of rubber fatigue: Configurational Mechanics and Critical Plane approaches. General theory of each approach was briefly presented. Two experimental data were considered: Saintier [36] and Mars [25]. Both predictors used in the present study were found to give good results in term of fatigue life prediction. Among different loading conditions, discrepancies between theoretical and measured fatigue live were mostly observed in the cases where torsion was predominant, i.e. simple torsion, alternating torsion, proportional compression/torsion. In such cases, the CMA-predictor appeared to give slightly better life predictions.

An important point to remember is the description of the constitutive behavior of the tested materials. As shown in this paper, for elastomers exhibiting Mullins effect such as Mars's material, the used of simple hyperelastic model such as Neo-Hookean requires careful consideration, particularly in the case of high strains complex loading conditions and complex specimens/structures where stress/strain gradients are present. Finally, to close the paper, it is to note that while the CPA-predictor was successfully implemented into the Finite Element Software, the numerical implementation of the CMA-predictor, particularly its increment over a fatigue cycle, is still under investigation. Therefore, further development is required in order to give more information concerning the efficiency of this predictor.

\section{References}

[1] Abraham F, Alshuth T, Jerrams S. The effect of minimum stress and stress amplitude on the fatigue life of non strain crystallising elastomers. Mater Design 2005;26:239-45
[2] Ait-Bachir M, Verron E, Mars W. Energy release rate of small cracks under finite multiaxial straining. In: 6th European conference on constitutive models for rubber (ECCMR); 2009.

[3] André N, Cailletaud G, Piques R. Haigh diagram for fatigue crack initiation prediction of natural rubber components. Kautsch Gummi Kunstst 1999;52:120-3.

[4] Andriyana A. Définition d'une nouvelle grandeur prédictive pour la durée de vie en fatigue des matériaux élastomères. PhD thesis, Ecole Centrale de Nantes, France; 2006.

[5] Andriyana A, Verron E. Prediction of fatigue life improvement in natural rubber using configurational stress. Int J Solids Struct 2007;44:2079-92.

[6] ASTM. Standard test method for rubber property - extension cyclic fatigue. ASTM D 4482-85; 1994.

[7] Cadwell M, Merril R, Sloman C, Yost F. Dynamic fatigue life of rubber. Indus Eng Chem 1940;12(1):19-23.

[8] Diani J, Fayolle B, Gilormini P. A review of the Mullins effect. Eur Polym J 2009;45(3):601-12.

[9] Eshelby JD. The force on an elastic singularity. Philos Trans R Soc Lond, A 1951;244:87-112.

[10] Eshelby JD. The elastic energy-momentum tensor. J Elasticity 1975;5(34):321-35.

[11] Fielding JH. Flex life and crystallization of synthetic rubber. Indus Eng Chem 1943;35:1259-61.

[12] Findley WN. A theory for the effect of mean stress on fatigue of metals under combined torsion and axial load or bending. J Eng Indus 1959:301-6.

[13] Gent AN. Science and technology of rubber. 2nd ed. New York: Academic Press; 1994. p. 471 [Chapter 10].

[14] Gent AN, Lindley PB, Thomas AG. Cut growth and fatigue of rubbers. I. The relationship between cut growth and fatigue. J Appl Polym Sci 1964;8:455-66.

[15] Green AE, Adkins JE. Large elastic deformations. Oxford: The Clarendon Press; 1960.

[16] Greensmith H. Rupture of rubber. X. The change in stored energy on making a small cut in a test piece held in simple extension. J Polym Sci 1963;7:993-1002.

[17] Holzapfel GA. Nonlinear solid mechanics. A continuum approach for engineering. Chichester: J. Wiley and Sons; 2000.

[18] Karolczuk A, Macha E. A review of critical plane orientations in multiaxial fatigue failure criteria of metallic materials. Int J Fract 2005;134:267-304.

[19] Kienzler R, Herrmann G. On the properties of the Eshelby tensor. Acta Mech 1997; 125:73-91.

[20] Lake GJ, Clapson BE. Truck tire groove cracking, theory and practice. Rubber Chem Technol 1971;44:1186-202.

[21] Le Cam JB. Endommagement en fatigue des élastomères. PhD thesis, Ecole Centrale de Nantes, France; 2005.

[22] Le Cam J-B, Huneau B, Verron E, Gornet L. Mechanism of fatigue crack growth in carbon black filled natural rubber. Macromolecules 2004;37:5011-7.

[23] Lindley P. Energy for crack growth in model rubber components. J Strain Anal 1972;7:132-40.

[24] Mandelkern L. Crystallization of polymers. Cambridge University Press; 2002.

[25] Mars WV. Multiaxial fatigue of rubber. PhD thesis, University of Toledo; 2001.

[26] Mars WV. Cracking energy density as a predictor of fatigue life under multiaxial conditions. Rubber Chem Technol 2002;75:1-17.

[27] Mars WV. Heuristic approach for approximating energy release rates of small cracks under finite strain, multiaxial loading. In: Covene V, editor. Elastomers and components - service life prediction: progress and challenges. PA: OCT Science; 2006. p. 89-109.

[28] Mars WV, Fatemi A. A literature survey on fatigue analysis approaches for rubber. Int J Fatigue 2002;24:949-61.

[29] Mars WV, Fatemi A. A novel specimen for investigating the mechanical behavior of elastomers under multiaxial loading conditions. Exp Mech 2004;44:136-46.

[30] Mars WV, Fatemi A. Observations of the constitutive response and characterization of filled natural rubber under monotonic and cyclic multiaxial. J Eng Mater Technol 2004;126:19-28.

[31] Maugin GA. Material inhomogeneities in elasticity. London: Chapman and Hall; 1993.

[32] Maugin GA. Material forces: concepts and applications. Appl Mech Rev $1995 ; 48(5)$.

[33] Ostoja-Kuczynski E. Comportement en fatigue des élastomères : application aux structures antivibratoires pour l'automobile. $\mathrm{PhD}$ thesis, Ecole Centrale de Nantes; 2005.

[34] Rivlin RS, Thomas AG. Rupture of rubber. I. Characteristic energy for tearing. J Polym Sci 1953;10:291-318.

[35] Roberts BJ, Benzies JB. The relationship between uniaxial and equibiaxial fatigue in gum and carbon black filled vulcanizates. In: Proceedings of Rubbercon'77, vol. 2; 1977. p. 2.1-13.

[36] Saintier N. Prévisions de la durée de vie en fatigue du NR sous chargement multiaxial. PhD thesis, Ecole de Mines de Paris, France; 2001.

[37] Saintier N, Cailletaud G, Piques R. Crack nucleation and propagation under multiaxial fatigue in a natural rubber. Int J Fatigue 2006;28:61-72.

[38] Saintier N, Cailletaud G, Piques R. Multiaxial fatigue life prediction for a natural rubber. Int J Fatigue 2006;28:530-9.

[39] Stanfield G. Discussion on The strength of metals under combined alternating stresses. In: Gough $\mathrm{H}$, Pollard $\mathrm{H}$, editors. Proceedings of the institution of mechanical engineers, vol. 131; 1935. p. 93. 
[40] Steinmann P. Application of material forces to hyperelastostatic fracture mechanics. I. Continuum mechanical setting. Int J Solids Struct 2000;37:7371-91.

[41] Toki S, Fujimaki T, Okuyama M. Strain-induced crystallization of natural rubber as detected real-time by wide-angle X-ray diffraction technique. Polymer 2000;41:5423-9.

[42] Trabelsi S, Albouy P, Rault J. Stress-induced crystallization properties of natural and synthetic cis-polyisoprene. Rubber Chem Technol 2003;77:303-16.

[43] Truesdell C, Noll W. Handbuch der Physik Bd. III/3: the nonlinear field theories of mechanics. Berlin: Springer-Verlag; 1965.
44] Verron E. Study of the simple extension tear test sample for rubber with configurational mechanics. Int J Fract 2007;147:219-25.

[45] Verron E, Ait-Bachir M, Castaing P. Some new properties of the Eshelby stress tensor. In: IUTAM symposium on progress in the theory and numerics of configurational mechanics. Friedrich-Alexander-Universitat, Germany; 2008.

[46] Verron E, Andriyana A. Definition of a new predictor for multiaxial fatigue crack nucleation in rubber. J Mech Phys Solids 2008;56:417-43.

[47] Verron E, Le Cam JB, Gornet L. A multiaxial criterion for crack nucleation in rubber. Mech Res Commun 2006;33:493-8.

[48] Young D. Application of fatigue methods based on fracture mechanics for tire compound development. Rubber Chem Technol 1990;63:567-81. 